\title{
Background Division, A Suitable Technique for Moving Object Detection
}

\author{
Walter Izquierdo-Guerra and Edel García-Reyes \\ Advanced Technologies Application Center (CENATAV), 7a $\sharp 21812$ e/ 218 y 222, \\ Rpto. Siboney, Playa, C.P. 12200, La Habana, Cuba \\ $\{$ wizquierdo, egarcia\}@cenatav.co.cu
}

\begin{abstract}
Nowadays, background model does not have any robust solution and constitutes one of the main problems in surveillance systems. Researchers are working in several approaches in order to get better background pixel models. This is a previous step to apply the background subtraction technique and results are not as good as expected. We concentrate our efforts on the second step for segmentation of moving objects and we propose background division to substitute background subtraction technique.This approach allows us to obtain clusters with lower intraclass variability and higher inter-class variability, this diminishes confusion between background and foreground,pixels. We compared results using our background division approach versus wallflowers algorithm [1] as the baseline to compare.
\end{abstract}

\section{Introduction}

Surveillance systems are interested in the problem of segmenting moving objects in video sequences. Background subtraction technique is one of the most widely used approaches. This algorithm compares the current image versus a background image obtained by a previous processing of the pixel history. Pixels where the difference is greater than a threshold are marked as foreground pixels. That is the main principle for this technique. In our opinion this kind of algorithms may be separate in two main steps: background maintenance and segmenting criteria.

The background maintenance is the step where the background is modeled. Next, an expected image according to his model is predicted. In general, this is the main feature that distinguishes methods. The current models report a lot of errors when predicting the background. Some researchers have produced state of the art of the existent methods in recent years [1], [2], [3] and [4].

The second step (segmenting criteria) has evolved since a simple priori threshold [5] to a more complex system as [1]. Some variables are inferred from the background maintenance phase in order to obtain an automatic threshold to segment foreground pixels. One of the most popular algorithms for moving object detection is the Gaussian mixture model. In [6], the authors explain a detailed version of it. At present, there are authors trying to improve this method because it has a great number of advantages. For example, the authors of [7] propose an 
approach that combines the Gaussian mixture model with a Markov random fields smoothness. That algorithm has a great computational cost. It fixes a lot of parameters. That turns the method into a scene-dependant method. A survey with a great amount of approaches can be found in $[8$. Most of them try to solve the problem of robust background maintenance, but the number of situations that can be observed in an image sequence is colossal.

In general, researchers have accepted the background subtraction technique as the basis of all the a posteriori processing. Our point is that background division is the most appropriate technique in order to do this work because it diminishes confusion between background and foreground pixels. This constitutes the second part of our work.

The main problems that affect the segmentation of moving objects are presented in [1]. We are going to focus our work on seven of them: moved object, time of day, light switch, waving trees, camouflage, bootstrap and foreground aperture. There are works which try to solve other problems. For example 9 shows an algorithm to solve the shadows in the image.

This paper is divided into 5 sections. Section 1 is an overview of this work. Section 2 describes the theoretical topics about the physical nature images. Section 3 presents our proposal of background division and explains why we prefer this technique. Section 4 shows the comparison of our results versus wallflowers algorithm and SACON's algorithm. Lastly, section 5 contains the conclusions.

\section{Fresnel Reflection}

The Fresnel equations describe the behavior of light when moving between mediums of different refractive indexes. The reflection of light that the equations predict is known as Fresnel reflection. When light moves from a medium of a

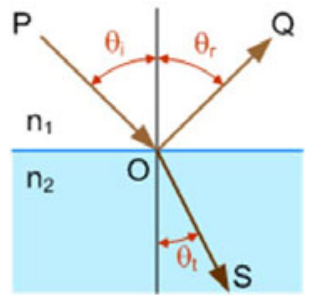

Fig. 1. Reflection and Refraction representation

given refractive index $n_{1}$ into a second medium with refractive index $n_{2}$, both reflection and refraction of the light may occur(see Figure 1). The angles of incident, reflected and refracted rays with respect to the normal of the surface that separate the mediums are given as $\Theta_{i}, \Theta_{r}$ and $\Theta_{t}$, respectively. Relationship among these angles is given by the law of reflection and Snell's law [10. The fraction of the incident energy that is reflected from the surface is given by the reflectance $R$ and the fraction that is refracted is given by the transmittance $T$, 
where $R=R\left(\Theta_{i}, n_{1}, n_{2}\right)$. In this way, the coefficient $R$ depends on the refraction indexes of the materials and the angle between the source and the object. We talk about Fresnel equations because it describes the theoretical behavior of the real world. In the next section we are going to observe how background subtraction and background division work for theoretical assumptions.

\section{Background Division}

As it was shown above, the coefficient $R$ depends on the incident angle and refractive indexes of the mediums. It means that we are going to work with an $R$ coefficient $R\left(\Theta_{i}, n_{1}, n_{2}, x, y\right)$ being $(x, y)$ the position of the pixel in the image. This is the physics behind the problem, but we are going to take into consideration some topics. In general, $\Theta_{i}$ can be variable(example the sun light). The sun is moving the entire day; but if we take into account small movement of the sun and the algorithm update speed, we can consider this source as fixed for small time intervals. In the case of indoor environments the light sources are fixed. Now we can ensure, that for our purposes, the reflection coefficient does not depend on $\Theta_{i}$.

$R$ also depends on the refraction indexes. In the case of $n_{1}$, it is always going to represent the air refraction index. In this way, it will be fixed in all the video. $n_{2}$ only changes if an object in the scene was moved(in this case $R$ changes too) and this is a situation we want to detect. Then we can characterize our scene by its reflectance. We are going to have one point $x, y$ with a reflectance value. But, we only observe the light intensity reflected by image $I$.

$$
I(x, y, t)=I_{o}(x, y, t) * R(x, y)
$$

where $t$ is the time and

$$
I_{o}(x, y, t)=I_{o}(t)+\epsilon(x, y, t)
$$

Where $I_{o}(t)$ is the proper source illumination. Here $\epsilon(x, y, t)$ is a natural noise that affects the three variables. It represents a gaussian distribution. So when we collect a big number of $\epsilon_{k}$ its expected value is 0 :

$$
\lim _{N \rightarrow \infty} \sum_{k}^{N} \epsilon_{k}(x, y, t)=0
$$

where $k=x, y$ or $t$. so,

$$
I(x, y, t)=\left[I_{o}(t)+\epsilon(x, y, t)\right] * R(x, y)
$$

Usually, the background $(B)$ is estimated as the expected value of $I(x, y, t)$ [1] [4. The are a lot of approaches to estimate the background. The most widely used are the mean value over $t$, median value, gaussian mixture models(GMM) 
among others. We are going to concentrate our efforts in the mean value in order to explain easily the problem.

$$
B(x, y)=\sum_{t=0}^{N} \frac{I(x, y, t)}{N}=\frac{R(x, y)}{N} *\left[\sum_{t=0}^{N} I_{o}(t)+\sum_{t=0}^{N} \epsilon_{t}\right]
$$

We consider $\mathrm{N}$ very big in order to suppress the noise. Then, according to equation 3 .

$$
\sum_{t=0}^{N} \epsilon_{t}=0
$$

So,

$$
B(x, y)=R(x, y) * \bar{I}_{o}
$$

where $\bar{I}_{o}$ is the mean value of $I_{o}(t)$

When a new frame is coming $(I(x, y, t+1))$ background subtraction technique is applied:

$$
S(x, y, t)=I(x, y, t+1)-B(x, y)=\left[I_{o}(t+1)+\epsilon(x, y, t+1)-\bar{I}_{o}\right] * R(x, y)
$$

Usually it is accept that the expected value of $S(x, y, t)$ is a matrix of zeros. This is only right when $I_{o}(t+1)=\bar{I}_{o}$ (An illumination change did not occur) and under certain conditions of $R(x, y)$. If background division technique is applied the following expression is obtained:

$$
\begin{gathered}
D(x, y, t)=\frac{I(x, y, t+1)}{B(x, y)}=\frac{I_{o}(t+1)+\epsilon(x, y, t+1)}{\bar{I}_{o}} * \frac{R(x, y)}{R(x, y)} \\
D(x, y, t)=\frac{I_{o}(t+1)}{\bar{I}_{o}}+\frac{\epsilon(x, y, t+1)}{\bar{I}_{o}}
\end{gathered}
$$

Notice that $D(x, y, t)$ maintains the original gaussian distribution. The expected value of $D(x, y, t)$ is:

$$
C(t)=\frac{I_{o}(t+1)}{\bar{I}_{o}}
$$

and the noise:

$$
\frac{\epsilon(x, y, t+1)}{\bar{I}_{o}}
$$

Computing the mean value over $x$ and $y$ of the matrix $D(x, y, t)$ (this is an observable magnitude) we obtain the value of $C(t)$.

When we use background division technique we obtain an expected value for the matrix $D(x, y)$ and we can use this value as a center of a gaussian, etc. This is the main advantage of background division. There are others, it is easy to predict the shadow and the magnitude of illumination change, as the mean of the divided image. But those topics are going to be discussed in future works.

As a conclusion, background subtraction is affected by the illumination change while background division is not affected. 
In order to apply our algorithm to the wallflower's dataset [1], for the modeling phase, we used the approximated median filter [11. This is a very simple method. In this way, we have a background image.

We have a second background to model the sudden illumination changes. It is used when the algorithm classifies more than 80 per cent of the pixels as foreground. This background is obtained in a training phase. 12

Now, when a new frame is coming we use the background to apply background division technique.

$$
D(x, y, t)=\frac{I(x, y, t+1)}{B(x, y)}
$$

In this way we are going to obtain a matrix $D(x, y, t)$ where certain value is predominant. Now, we consider there is not only one predominant value, we are going to suppose there are 5 most probably values in order to classify all pixels in the image. We apply $\mathrm{k}$-means algorithm to find this 5 values. We initialize with the seeds $\left(\begin{array}{lllll}0 & 0.8 & 1 & 1.2\end{array}\right)$. The value near 1 is a background value. The other four clusters are going to be classified as foreground.

We also take into account another advantage of the method. When we have shadows in the scene, these are poorer illuminated places than the rest of the image. When we apply background division, we can predict this shadows as values minor than 1 but near to it.

In our paper we classify a second cluster as background if it is close enough to the cluster centered on 1 .

The processing continues applying a connected components algorithm. We keep the regions greater or equal than a size estimated for a person (suitable for each scene).

We apply a segmenting criteria similar to the explained in [12]:

Let $A(x, y)$ be the binary image at this point of processing.

We compute the distant transform $(d(x, y))$ of $A[13]$. We are going to classify a pixel as foreground in $F(x, y)$ only if $D_{t}(x, y) * d_{t}(x, y)<\tau$.

Where $F(x, y)$ is the final binary image that our algorithm return.

\section{Results}

We compare our results versus wallflower's results [1. Wallflower's algorithm is a very famous method and its dataset is one of the most widely used to compare algorithms. We compare our results vs. 12] results too. It is an algorithm where background subtraction technique is applied.

The visual results are shown in figure 2 and error rates are shown on Table 1.

As we can observe in Table 1, our algorithm works better than the wallflower algorithm. It reduces to $56 \%$ the total of errors they reported for background subtraction and to $53 \%$ for background division.

In light switch image, there is a great amount of false negative pixels. In our opinion, this does not constitute an error of our algorithm because most of them are pixels belonging to a chair, that wallflower dataset reports as an object. We consider that the chair is background in the scene. 


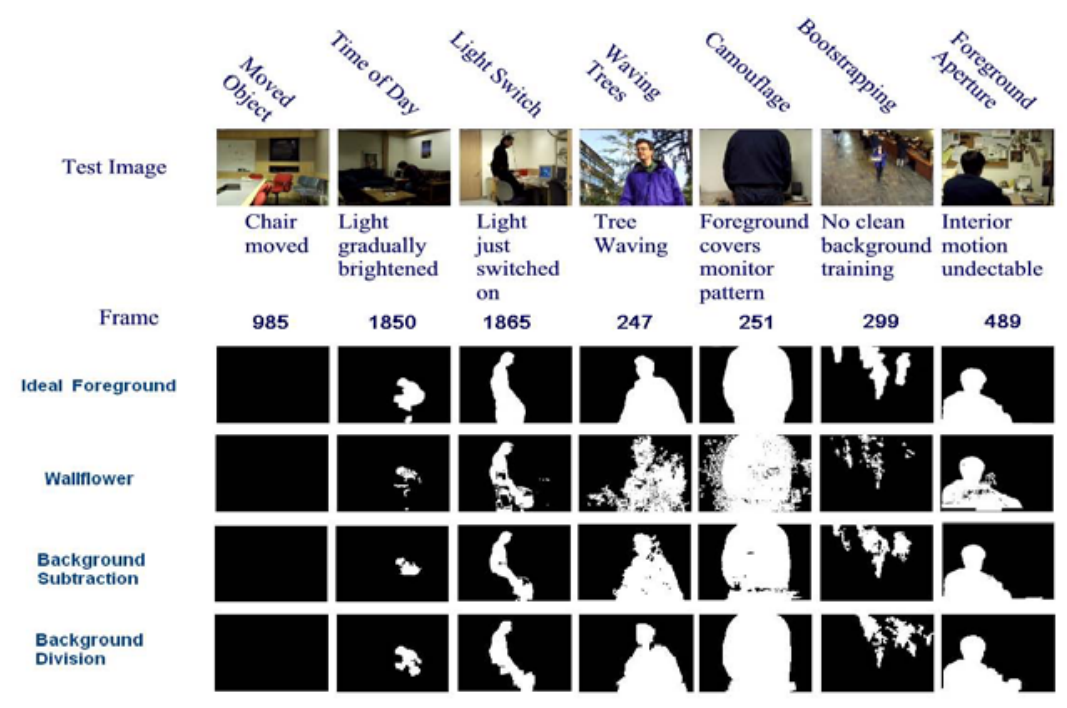

Fig. 2. Visual results. The first row of pictures is hand-segmented images.

Table 1. Numeric results

\begin{tabular}{|c|c|c|c|c|c|c|c|c|c|}
\hline Algorithm & $\begin{array}{l}\text { Error } \\
\text { Type }\end{array}$ & $\begin{array}{l}\text { moved } \\
\text { object }\end{array}$ & time & \begin{tabular}{|l|} 
light \\
switch
\end{tabular} & \begin{tabular}{|c|} 
waving \\
trees
\end{tabular} & amouflage & bootstrap & $\begin{array}{c}\text { foreground } \\
\text { aperture }\end{array}$ & $\begin{array}{l}\text { Total } \\
\text { Errors }\end{array}$ \\
\hline \multirow{2}{*}{ Wallflower[1] } & false neg. & 0 & 961 & 947 & 877 & 229 & 2025 & 320 & \multirow{2}{*}{11448} \\
\hline & \begin{tabular}{|l} 
false pos. \\
.
\end{tabular} & 0 & 25 & 345 & 1999 & 2706 & 365 & 649 & \\
\hline \multirow{2}{*}{ Background subtraction 12} & false neg. & 0 & 1030 & 1308 & 164 & 518 & 907 & 236 & \multirow{2}{*}{5906} \\
\hline & false pos. & 0 & 3 & 385 & 333 & 384 & 565 & 73 & \\
\hline \multirow{2}{*}{ Background division } & false neg. & 0 & 638 & 1059 & 108 & 164 & 1287 & 152 & \multirow{2}{*}{5579} \\
\hline & false pos. & 0 & 64 & 417 & 192 & 379 & 833 & 286 & \\
\hline
\end{tabular}

It is possible to see that the noise in the silhouettes is lower for background division. However, a small trend to rise the false positive rate was observed, especially with bootstrapping problem. The advantage of background division is that interclass variability is lower than for background subtraction, then the confusion between background and foreground pixel is lower. This way a clustering algorithm may separate moving objects and shadows in different connected components.

\section{Conclusions}

In this paper, we present a novel approach to the moving object detection problem. We use the background division approach to obtain a model suitable to illumination changes. The global threshold used to segment the moving objects is dependent on the current image noise level and it is automatically calculated applying an empirical formula 12 . 
We experimentally compared our approach against the wallflower algorithm and we obtained better results, as showed visually in figure 2, and numerically in Table 1. Our future research direction is to combine our algorithm with a more robust tool to model the pixel history.

On the other hand, to the best of our knowledge, this is the first time approaches of background subtraction and background division for segmentation of moving object have been compared taking into account different problems affecting the segmentation. In general, for the wallflower dataset, the background division outperforms background subtraction, especially when the light gradually changes. This was visual and numerically observed in the experiments, and the total error rate was better than previous published results.

\section{References}

1. Toyama, K., Krumm, J., Brumitt, B., Meyers, B.: Wallower: Principles and practice of background maintenance. In: Seventh International Conference on Computer Vision, vol. 1, p. 255 (1999)

2. Sen, Kamath, C.: Robust techniques for background subtraction in urban traffic video, vol. 5308, pp. 881-892. SPIE, San Jose (2004)

3. Ribeiro, H.N., Hall, D., Nascimento, J., Ribeiro, P., Andrade, E., Moreno, P., Pesnel, S., List, T., Emonet, R., Fisher, R.B., Santos Victor, J., Crowley, J.L.: Comparison of target detection algorithms using adaptive background models. In: Proc. 2nd Joint IEEE Int. Workshop on Visual Surveillance and VisLab-TR 13/2005, pp. 113-120 (2005)

4. Mcivor, A.M.: Background subtraction techniques. In: Proc. of Image and Vision Computing, pp. 147-153 (2000)

5. Heikkilä, J., Silvén, O.: A real-time system for monitoring of cyclists and pedestrians. Image and Vision Computing 22(7), 563-570 (2004)

6. Power, P.W., Schoonees, J.A.: Understanding background mixture models for foreground segmentation. In: Proceedings Image and Vision Computing, New Zealand, p. $267(2002)$

7. Schindler, K., Wang, H.: Smooth foreground-background segmentation for video processing (2006)

8. Bouwmans, T., Baf, F.E., Vachon, B.: Background modeling using mixture of gaussians for foreground detection - a survey. Recent Patents on Computer Science 1, 219-237 (2008)

9. Kaewtrakulpong, P., Bowden, R.: An improved adaptive background mixture model for realtime tracking with shadow detection. In: Proc. 2nd European Workshop on Advanced Video Based Surveillance Systems, AVBS 2001, VIDEO BASED SURVEILLANCE SYSTEMS: Computer Vision and Distributed Processing (2001)

10. Frish, S., Timoreva, A.: Curso de Física General, vol. 3. MIR, Moscow (1973)

11. Bécsi And, T., Péter, T.: A mixture of distributions background model for traffic video surveillance. Periodica Polytechnica Ser. Transp. Eng. 34, 109-117 (2006)

12. Izquierdo-Guerra, W., García-Reyes, E.B.: A novel approach to robust background subtraction. In: Bayro-Corrochano, E., Eklundh, J.-O. (eds.) CIARP 2009. LNCS, vol. 5856, pp. 69-76. Springer, Heidelberg (2009)

13. Gonzalez, R.C., Woods, R.E., Eddins, S.L.: Digital Image Processing Using MATLAB. Prentice-Hall, Inc., Upper Saddle River (2003) 